JIRSS (2018)

Vol. 17, No. 02, pp 57-89

DOI: $10.29252 /$ jirss.17.2.5

\title{
On Modified Log Burr XII Distribution
}

\author{
Fiaz A. Bhatti ${ }^{1}$, G.G. Hamedani ${ }^{2}$ and Munir Ahmad ${ }^{1}$ \\ ${ }^{1}$ National College of Business Administration and Economics, Lahore, Pakistan. \\ ${ }^{2}$ Department of Mathematics, Statistics and Computer Science, Marquette University, Milwau- \\ kee, USA.
}

Received: 17/05/2017, Revision received: 07/12/2017, Published online: 08/08/2018

\begin{abstract}
In this paper, we present a modified Log Burr XII (MLBXII) distribution developed on the basis of a generalized log Pearson differential equation. This distribution is also obtained from a compounding mixture of distributions. Moments, inequality measures, uncertainty measures and reliability measures are theoretically established. Characterizations of MLBXII distribution are also studied via different techniques. Parameters of MLBXII distribution are estimated using the maximum likelihood method. Goodness of fit of this distribution through different methods is studied.
\end{abstract}

Keywords. Characterizations, Maximum likelihood estimation, Moments, Reliability, Uncertainty.

MSC: 60E07; 60E10; 62E10; 62E20.

Fiaz A. Bhatti (fiazahmad72@gmail.com)

Corresponding Author: G.G. Hamedani (g.hamedani@mu.edu)

Munir Ahmad (munirahmaddr@yahoo.co.uk) 


\section{Introduction}

Burr (1942) suggested 12 distributions as the Burr family to fit cumulative frequency functions on frequency data. The Burr XII (BXII) distribution is widely used in modeling lifetimes, financial and insurance data. BXII distributions also have wide applications in the reliability and acceptance sampling plans. In many areas of applications, the Burr distribution of any type is inadequate and as such various generalizations and extensions of the Burr distribution have been proposed in the statistical literature.

The cumulative distribution function (cdf) for random variable $Y$ having a BXII distribution is given by

$$
G(y)=1-\left(1+y^{\beta}\right)^{-\alpha}, \quad y \geq 0, \alpha>0, \beta>0 .
$$

The probability density function (pdf) of a BXII distribution for the random variable $Y$ is

$$
g(y)=\alpha \beta y^{(\beta-1)}\left(1+y^{\beta}\right)^{-(\alpha+1)}, \quad y>0, \alpha>0, \beta>0 .
$$

Recently, new generated families of continuous distributions have attracted a good number of statisticians to develop new models. These families are obtained by introducing one or more additional threshold/shape/scale parameter(s) or using some transformation to the baseline distribution.

Many authors, like Zimmer and Burr (1963), Takahasi (1965), Tadikamalla (1980), Saran and Pushkarna (1999), Begum and Parvin (2002), Shao et al. (2004), Olapade (2008), Paranaiba et al. (2011), Usta (2013) and Paranaiba et al. (2013), studied BXII distribution. Okasha et al. (2015) studied BXII distributions and their various properties. Silva and Cordeiro (2015) developed a new compounding family by mixing BXII and power series distributions. Gomes et al. (2015) presented McDonald Burr XII distributions along with properties and applications. Muhammad (2016) established the generalized BXII- Poisson distribution to analyze the strength of materials. Afify et al. (2016) considered Weibull BXII distributions for analyzing the strengths of glass fibers. Thupeng (2016) modeled concentrations of the daily extreme nitrogen dioxide with BXII distributions.

Dogrru and Arslan (2016) estimated the parameters of BXII distributions with optimal B-robust estimators. Yari and Tondpour (2017) derived a new Burr distribution to study the lifetime cancer data. Ghosh and Bourguignon (2017) presented properties of the extended BXII distributions. Mdlongwa et al. (2017) studied the properties and applications of BXII modified Weibull distributions. Mead and Afify (2017) also presented properties and applications of BXII distributions with five parameters. Bhatti 
et al. (2018) have studied the generalized log Pearson differential equation (GLPE) to develop probability distributions with various choices of the coefficients. Guerra et al. (2017) developed a gamma burr XII distribution with a flexible hazard function and studied its various properties and applications. Cadena (2017) studied different behaviors of the extended Burr XII distribution. Kumar (2017) studied different properties of the Burr type XII distribution.

In this paper, a new family of distributions is proposed, that is a more flexible probability model in fitting lifetime data, annual maxima of river flows, strengths of materials, fracture toughness data, and describing special types of hazard functions. This family of distributions provides better fits for strengths measured in GPA for single fibers than sub and other competing models. The rest of the article is composed of the following sections. In Section 2, the pdf for a MLBXII distribution is developed on the basis of the generalized log Pearson differential equation (GLPE). In Section 3, MLBXII distribution is studied in terms of some plots and some basic structural properties. In Section 4, moments, negative moments, central moments, incomplete moments, L-moments, TL-moments, inequality measures, residual life functions and some other properties are presented. The reliability measures and uncertainty measures are studied in Section 5. In Section 6, MLBXII distribution is obtained from some compound scale mixture of the generalized log Weibull (GLW) distribution and gamma and size-biased Erlang distributions. In Section 7, characterizations of MLBXII distributions are studied based on (i) Truncated moment of the log of a random variable (ii) Truncated moment of a function (not log) of a random variable (iii) The hazard function (iv) Mills ratios (v) certain functions of the random variable (vi) the distribution of the $1^{\text {st }}$ order statistic, and (vii) the conditional expectation of record values. In Section 8, the potentiality of MLBXII distributions is illustrated by their application to a real data set and parameters of MLBXII are estimated using the maximum likelihood method. Goodness of fit of the probability distribution through different methods is also studied in this section. Finally, in Section 9, we provide some concluding remarks.

\section{Development of MLBXII Distribution}

In this section, we derive the pdf for MLBXII distributions using the generalized log Pearson differential equation as

$$
d[\ln f(x)]=\frac{1}{x} \frac{a_{0}+a_{1}(\ln x)+a_{2}(\ln x)^{2}+\ldots+a_{m}(\ln x)^{m}}{b_{0}+b_{1}(\ln x)+b_{2}(\ln x)^{2}+\ldots+b_{n}(\ln x)^{n}} d x, \quad x>1, m, n=1,2 \ldots
$$


Taking $a_{2}=a_{3}=\cdots=a_{m-2}=0, b_{0}=0, b_{2}=b_{3}=\cdots=b_{n-1}=0, a_{1}=-b_{1}, a_{m}=-b_{n}$ and $m=n=a+1$, we have

$$
\frac{d}{d x}[\ln f]=\frac{1}{x}\left\{\frac{a_{0}+a_{1}(\ln x)+a_{m-1}(\ln x)^{a}+a_{m}(\ln x)^{a+1}}{b_{1}(\ln x)+b_{n}(\ln x)^{a+1}}\right\}, \quad x>1 .
$$

After simplification and integration of both sides of (2.2), we obtain

$$
f(x)=k\left(\frac{1}{x}\right)\left(\frac{\ln x}{\left(b_{1}\right)^{\frac{1}{a}}}\right)^{\frac{a_{0}}{b_{1}}}\left(1+\frac{b_{n}}{b_{1}}(\ln x)^{a}\right)^{-\left(\frac{a_{0} b_{n}}{a b_{n} b_{1}}-\frac{a_{m-1}}{a b_{n}}\right)} .
$$

The normalizing constant $k$ is

$$
k=\frac{a\left(b_{1}\right)^{\frac{a_{0}}{a b_{1}}}}{\left(\frac{b_{1}}{b_{n}}\right)^{\frac{a_{0}}{a b_{1}}+\frac{1}{a}} B\left(\frac{a_{0}}{a b_{1}}+\frac{1}{a},-\frac{a_{m-1}}{a b_{n}}-\frac{1}{a}\right)},
$$

with $a_{m-1}<0$, where $B($.$) is the beta function. Taking a_{0}=(a-1), a_{1}=-1, a_{m-1}=$ $-(a p+c), a_{m}=-c, b_{1}=1, b_{n}=c$, we obtain the pdf of MLBXII distributions with parameters $a, c$ and $p$ as

$$
f(x)=\frac{a p}{x}(\ln x)^{a-1}\left[1+c(\ln x)^{a}\right]^{-\left(\frac{p}{c}+1\right)}, \quad x>1, a>0, c>0, p>0 .
$$

The cdf of MLBXII distributions with parameters $a, c$ and $p$ is given by

$$
F(x)=1-\left[1+c(\ln x)^{a}\right]^{-\frac{p}{c}}, \quad x \geq 1, a>0, c>0, p>0 .
$$

\section{Mathematical Properties of MLBXII Distribution}

MLBXII distributions are studied in terms of some plots and some basic structural properties. Sub-models of MLBXII distributions are also studied.

\subsection{Basic Structural Properties}

The survival function of MLBXII distribution with parameters $a, c$ and $p$ is

$$
S(x)=\left[1+c(\ln x)^{a}\right]^{-\frac{p}{c}}, \quad x \geq 1 .
$$




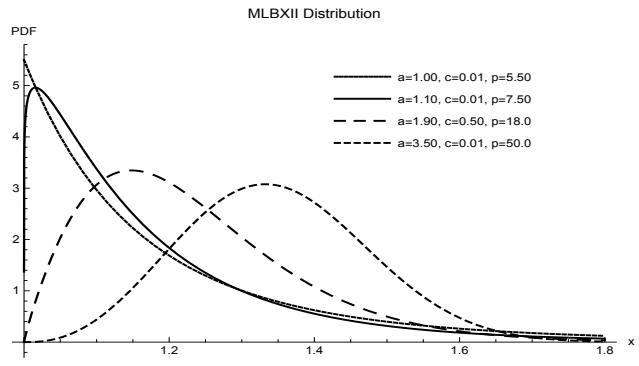

(a) Figure 1: Plots of pdf.

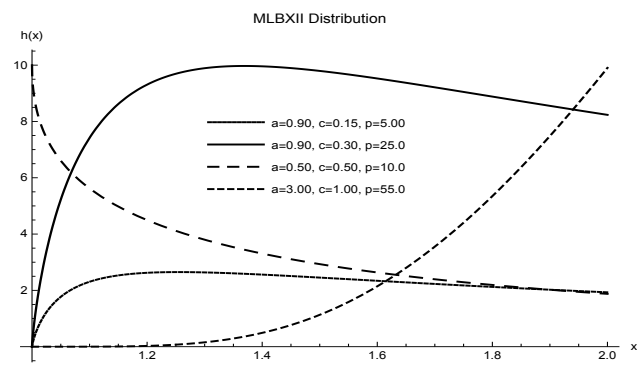

(b) Figure 2: Plots of hazard rate function.

As $x$ increases, the survival function has a reverse $J$-shape.

The hazard rate function of the random variable $X$ with a MLBXII distribution is

$$
h(x)=\frac{a p}{x}(\ln x)^{a-1}\left[1+c(\ln x)^{a}\right]^{-1}, \quad x>1 .
$$

The graphs show that MLBXII distributions are arc-shaped, exponential, positively skewed and symmetrical. The hazard rate function of MLBXII distributions is increasing, decreasing and constant after some time steps.

The cumulative hazard function of MLBXII distributions is

$$
H(x)=\int_{1}^{x} \frac{f(u)}{S(u)} d u=\frac{p}{c} \ln \left[1+c(\ln x)^{a}\right], \quad x>1 .
$$

The cumulative probability of the failure per unit of distance, time or cycles is the units of $H(x)$. The reverse hazard function of MLBXII distribution is

$$
r(x)=\frac{\frac{a p}{x}(\ln x)^{a-1}\left[1+c(\ln x)^{a}\right]^{-\left(\frac{p}{c}+1\right)}}{1-\left[1+c(\ln x)^{a}\right]^{-\frac{p}{c}}}, \quad x>1 .
$$

The Mills ratio of a MLBXII distribution is

$$
m(x)=\frac{x}{a p}(\ln x)^{-a+1}\left[1+c(\ln x)^{a}\right], \quad x>1 .
$$

The elasticity $e(x)=\frac{d \ln F(x)}{d \ln x}=x r(x)$ of a MLBXII distribution is given by

$$
e(x)=a p(\ln x)^{a-1}\left[1+c(\ln x)^{a}\right]^{-\left(\frac{p}{c}+1\right)}\left(1-\left[1+c(\ln x)^{a}\right]^{-\frac{p}{c}}\right)^{-1}, \quad x>1 .
$$



0, i.e.,

The mode $\widehat{x}$ of a MLBXII distribution with pdf (2.5) is obtained by solving $\frac{d}{d x}(\ln (f(x)))=$

$$
c(\ln x)^{a+1}+(a p+c)(\ln x)^{a}+(\ln x)-(a-1)=0 .
$$

For $a=1$, we obtain $\widehat{x}=\exp \left(-\frac{(p+1+c)}{c}\right)$.

\subsection{Quantile function of MLBXII Distributions}

The quantile function of a MLBXII distribution is

$$
x_{q}=\exp \left(c^{-\frac{1}{a}}\left[(1-q)^{-\frac{c}{p}}-1\right]^{\frac{1}{a}}\right)
$$

For $q=\frac{1}{2}$, the median of MLBXII distribution is

$$
\text { Median }=\exp \left(c^{-\frac{1}{a}}\left[2^{\frac{c}{p}}-1\right]^{\frac{1}{a}}\right)
$$

Also, the random number generator for MLBXII distribution is

$$
X=\exp \left(c^{-\frac{1}{a}}\left[(1-Z)^{-\frac{c}{p}}-1\right]^{\frac{1}{a}}\right)
$$

where the random variable $Z$ has the uniform distribution on $(0,1)$.

\subsection{Sub Models of MLBXII Distribution}

(i) For $p=1$, a MLBXII distribution reduces to a new modified log Fisk distribution.

(ii) For $p=1, c=1$, a MLBXII distribution reduces to a new log Fisk distribution.

(iii) For $a=p, c=1$, a MLBXII distribution reduces to a new log Para-logistic distribution.

(iv) For $a=1, p=1$, a MLBXII distribution reduces to a new log inverse Pareto distribution.

(v) For $a=\frac{1}{c}, p=1$, a MLBXII distribution reduces to a new $\log$ Pareto-III distribution.

(vi) For $c=1$, a MLBXII distribution reduces to a new log Burr XII (LBXII) distribution. 
(vii) For $a=1$, a MLBXII distribution reduces to a new modified log Lomax distribution.

(viii) For $a=1, c=1$, a MLBXII distribution reduces to a new $\log$ Lomax distribution.

(ix) For $c \rightarrow 0$, a MLBXII distribution reduces to a new $\log$ Weibull distribution.

(x) For $c \rightarrow 0, a=1$, a MLBXII distribution reduces to a new log exponential distribution.

(xi) For $\ln X=Y$, a MLBXII distribution reduces to a modified BXII distribution.

(xii) For $\ln X=Y, c=1$, a MLBXII distribution reduces to a BXII distribution.

(xiii) For $\ln X=Y, a=1$, a MLBXII distribution reduces to a new modified Lomax distribution.

(xiv) For $\ln X=Y, a=1, c=1$, a MLBXII distribution reduces to Lomax distribution.

\section{Moments}

In this section, moments, negative moments, central moments, incomplete moments, inequality measures, residual life functions and some other properties are presented.

\subsection{Moments of MLBXII Distribution}

The moments of $X$ with a MLBXII distribution about the origin are given by

$$
\mu_{r}^{\prime}=E\left(X^{r}\right)=\int_{1}^{\infty} x^{r} f(x) d x=\int_{1}^{\infty} x^{r} \frac{a p}{x}(\ln x)^{a-1}\left(1+c(\ln x)^{a}\right)^{-\left(\frac{p}{c}+1\right)} d x .
$$

Let $c(\ln x)^{a}=z$. Then, we have

$$
\begin{aligned}
E\left(X^{r}\right) & =\frac{p}{c} \int_{0}^{\infty} e^{r c^{-\frac{1}{a}} z^{\frac{1}{a}}}[1+z]^{-\left(\frac{p}{c}+1\right)} d z \\
& =p c^{-1} \sum_{i=0}^{\infty} \frac{c^{-\frac{i}{a}} r^{i}}{i !} \int_{0}^{\infty} z^{\frac{i}{a}}[1+z]^{-\left(\frac{p}{c}+1\right)} d z \\
& =p \sum_{i=0}^{\infty} \frac{r^{i}}{i !} d_{i}(a, c, p), \quad r=1,2,3, \ldots,
\end{aligned}
$$


where $d_{i}(a, c, p)=c^{-\frac{(a+i)}{a}} B\left(\frac{i}{a}+1, \frac{p}{c}-\frac{i}{a}\right)$ with $\frac{p}{c}>\frac{r}{a}$.

Mean and Variance of a MLBXII distribution are

$$
\begin{aligned}
E(X) & =p \sum_{i=0}^{\infty} \frac{d_{i}(a, c, p)}{i !}, \\
\operatorname{Var}(X) & =p \sum_{i=0}^{\infty} \frac{(2)^{i}}{i !} d_{i}(a, c, p)-\left(p \sum_{i=0}^{\infty} \frac{d_{i}(a, c, p)}{i !}\right)^{2} .
\end{aligned}
$$

An important measure of variability of a MLBXII random variable, known as Fisher index of dispersion, is

$$
F I=\frac{\operatorname{Var}(X)}{E(X)}=\frac{p \sum_{i=0}^{\infty} \frac{(2)^{i} d_{i}(a, c, p)}{i !}-\left(p \sum_{i=0}^{\infty} \frac{d_{i}(a, c, p)}{i !}\right)^{2}}{p \sum_{i=0}^{\infty} \frac{d_{i}(a, c, p)}{i !}} .
$$

For $F I=1$, MLBXII distributions are equidispersed; for $F I<1$, MLBXII distributions are under dispersed and for $F I>1$, MLBXII distributions are over dispersed.

The fractional positive moments of $X$ with s MLBXII distribution are

$$
\mu_{\frac{m}{n}}^{\prime}=E\left(X^{\frac{m}{n}}\right)=p \sum_{i=1}^{\infty} \frac{\left(\frac{m}{n}\right)^{i} d_{i}(a, c, p)}{i !} .
$$

The $r^{\text {th }}$ negative moment about the origin of $X$ with a MLBXII distribution is

$$
\mu_{-r}^{\prime}=E\left(X^{-r}\right)=\int_{1}^{\infty} x^{-r} \frac{a p}{x}(\ln x)^{a-1}\left(1+c(\ln x)^{a}\right)^{-\left(\frac{p}{c}+1\right)} d x .
$$

Let $c(\ln x)^{a}=z$. Then, we have

$$
\begin{aligned}
E\left(X^{-r}\right) & =\frac{p}{c} \int_{0}^{\infty} e^{-r c^{-\frac{1}{a}} z^{\frac{1}{a}}}[1+z]^{-\left(\frac{p}{c}+1\right)} d z \\
& =p c^{-1} \sum_{i=0}^{\infty} \frac{c^{-\frac{i}{a}}(-r)^{i}}{i !} \int_{0}^{\infty} z^{\frac{i}{a}}[1+z]^{-\left(\frac{p}{c}+1\right)} d z \\
& =p \sum_{i=1}^{\infty} \frac{(-r)^{i}}{i !} d_{i}(a, c, p) .
\end{aligned}
$$


Negative moments are helpful in harmonic means and many other measures.

The fractional negative moment of $X$ with MLBXII distribution is

$$
\mu_{-\frac{m}{n}}^{\prime}=E\left(X^{-\frac{m}{n}}\right)=p \sum_{i=1}^{\infty} \frac{\left(-\frac{m}{n}\right)^{i} d_{i}(a, c, p)}{i !} .
$$

The $k^{\text {th }}$ moment about the mean of $X$ is determined from the relationship

$$
\mu_{k}=E[X-E(X)]^{k}=\sum_{j=1}^{k}\left(\begin{array}{l}
k \\
j
\end{array}\right)(-1)^{j} \mu_{j}^{\prime} \mu_{(k-j)}^{\prime} .
$$

The $k^{\text {th }}$ moment about the mean of $X$ with a MLBXII distribution is determined from the relationship

$$
\mu_{k}=\sum_{j=1}^{k}\left(\begin{array}{l}
k \\
j
\end{array}\right)(-1)^{j}\left[p \sum_{i=0}^{\infty} \frac{(j)^{i}}{i !} d_{i}(a, c, p)\right]\left[p \sum_{i=0}^{\infty} \frac{((k-j))^{i}}{i !} d_{i}(a, c, p)\right] .
$$

Pearson's measure of skewness $\gamma_{1}$ is given by $\gamma_{1}=\frac{\mu_{3}}{\left(\mu_{2}\right)^{\frac{3}{2}}}$. Equivalently,

$$
\gamma_{1}=\frac{\sum_{j=1}^{3}\left(\begin{array}{l}
3 \\
j
\end{array}\right)(-1)^{j}\left[p \sum_{i=0}^{\infty} \frac{(j)^{i}}{i !} d_{i}(a, c, p)\right]\left[p \sum_{i=0}^{\infty} \frac{(3-j)^{i}}{i !} d_{i}(a, c, p)\right]}{\left(\sum_{j=1}^{2}\left(\begin{array}{c}
2 \\
j
\end{array}\right)(-1)^{j}\left[p \sum_{i=0}^{\infty} \frac{(j)^{i}}{i !} d_{i}(a, c, p)\right]\left[p \sum_{i=0}^{\infty} \frac{((2-j))^{i}}{i !} d_{i}(a, c, p)\right]\right)^{\frac{3}{2}}} .
$$

Pearson measure of Kurtosis $\beta_{2}$ is given by $\beta_{2}=\frac{\mu_{4}}{\left(\mu_{2}\right)^{2}}$. Equivalently,

$$
\beta_{2}=\frac{\sum_{j=1}^{4}\left(\begin{array}{l}
4 \\
j
\end{array}\right)(-1)^{j}\left[p \sum_{i=0}^{\infty} \frac{(j)^{i}}{i !} d_{i}(a, c, p)\right]\left[p \sum_{i=0}^{\infty} \frac{((4-j))^{i}}{i !} d_{i}(a, c, p)\right]}{\left(\sum_{j=1}^{2}\left(\begin{array}{l}
2 \\
j
\end{array}\right)(-1)^{j}\left[p \sum_{i=0}^{\infty} \frac{(j)^{i}}{i !} d_{i}(a, c, p)\right]\left[p \sum_{i=0}^{\infty} \frac{((2-j))^{i}}{i !} d_{i}(a, c, p)\right]\right)^{2}} .
$$

The moment generating function for the random variable $X$ with a MLBXII distribution is

$$
M_{X}(t)=E\left[e^{t X}\right]=\sum_{r=1}^{\infty} \frac{t^{r}}{r !} E(X)^{r}=\sum_{r=1}^{\infty} \frac{t^{r}}{r !} \mu_{r}^{\prime}=p \sum_{r=1}^{\infty} \sum_{i=0}^{\infty} \frac{t^{r}}{r !} \frac{(r)^{i}}{i !} d_{i}(a, c, p) .
$$


The cumulants of a MLBXII distribution for the random variable $X$ obtained from the recurrence relation $k_{r}=E\left(X^{r}\right)-\sum_{c=1}^{r-1}\left(\begin{array}{l}r-1 \\ c-1\end{array}\right) k_{c} E\left(X^{r-c}\right)$ are

$$
k_{r}=p \sum_{i=0}^{\infty} \frac{r^{i} d_{i}(a, c, p)}{i !}-p \sum_{c=1}^{r-1}\left(\begin{array}{c}
r-1 \\
c-1
\end{array}\right) k_{c} \sum_{i=0}^{\infty} \frac{(r-c)^{i}}{i !} d_{i}(a, c, p) .
$$

The $r^{\text {th }}$ moment (about the origin) of $\ln (X)$ with a MLBXII distribution is

$$
E\left[(\ln (X))^{r}\right]=\int_{1}^{\infty}(\ln x)^{r} \frac{a p}{x}(\ln x)^{a-1}\left(1+c(\ln x)^{a}\right)^{-\left(\frac{p}{c}+1\right)} d x
$$

Let $c(\ln x)^{a}=z$. Then, we have

$$
E\left[(\ln (X))^{r}\right]=p c^{-\frac{(a+r)}{a}} B\left(\frac{r}{a}+1, \frac{p}{c}-\frac{r}{a}\right), \quad \text { with } \quad \frac{p}{c}>\frac{r}{a}, r=1,2,3, \ldots .
$$

The factorial moments of a MLBXII distribution are given by

$$
\begin{aligned}
E[X]_{n} & =\sum_{r=1}^{n} \alpha_{r} E\left(X^{r}\right) \\
& =\sum_{r=1}^{n} \alpha_{r} \mu_{r}^{\prime}=\sum_{r=1}^{n}\left[\alpha_{r} p \sum_{i=0}^{\infty} \frac{r^{i}}{i !} d_{i}(a, c, p)\right],
\end{aligned}
$$

where $[X]_{i}=X(X+1)(X+2) \ldots(X+i-1)$ and $\alpha_{r}$ is Stirling number of the first kind.

\subsection{The Mellin Transform of MLBXII Distribution}

The Mellin transform helps to determine moments for a probability distribution. By definition, Mellin transform is

$$
\mathrm{M}\{f(x) ; k\}=f^{*}(k)=\int_{0}^{\infty} f(x) x^{k-1} d x .
$$

The Mellin transform of $X$ with a MLBXII distribution is written as

$$
\begin{aligned}
\mathrm{M}\{f(x) ; k\} & =\int_{1}^{\infty} f(x) x^{k-1} d x \\
& =\int_{1}^{\infty} x^{k-1} \frac{a p}{x}(\ln x)^{a-1}\left(1+c(\ln x)^{a}\right)^{-\left(\frac{p}{c}+1\right)} d x \\
& =p \sum_{i=1}^{\infty} \frac{(k-1)^{i}}{i !} d_{i}(a, c, p) .
\end{aligned}
$$




\subsection{Moments of Order Statistics}

Moments of order statistics are used to predict the failure of future items based on the times of a few early failures. The pdf for $m^{\text {th }}$ order statistic $X_{m: n}$ is

$$
f\left(x_{m: n}\right)=\frac{1}{B(m, n-m+1)}[F(x)]^{m-1}[1-F(x)]^{n-m} f(x) .
$$

The pdf of $X_{m: n}$ for a MLBXII distribution is

$$
f\left(x_{m: n}\right)=\frac{\sum_{j=0}^{m-1}(-1)^{j}\left(\begin{array}{l}
m-1 \\
j
\end{array}\right)}{B(m, n-m+1)} \frac{a p}{x}(\ln x)^{a-1}\left[1+c(\ln x)^{a}\right]^{-\frac{p}{c}(n-m+j+1)-1} .
$$

The $r^{\text {th }}$ moments of $X_{m: n}$ (about the origin) is given by

$$
\begin{aligned}
E\left(X_{m: n}^{r}\right)= & \int_{1}^{\infty} x^{r} f\left(x_{m: n}\right) d x \\
& =\int_{1}^{\infty} x^{r} \frac{\sum_{j=0}^{m-1}(-1)^{j}\left(\begin{array}{l}
m-1 \\
j
\end{array}\right)}{B(m, n-m+1)} \frac{a p}{x}(\ln x)^{a-1}\left[1+c(\ln x)^{a}\right]^{-\frac{p}{c}(n-m+j+1)-1} d x \\
& =\frac{\sum_{j=0}^{m-1}(-1)^{j}\left(\begin{array}{l}
m-1 \\
j
\end{array}\right)}{B(m, n-m+1)} p \sum_{i=0}^{\infty} \frac{r^{i} c^{-\frac{(a+i)}{a}}}{i !} B\left(\frac{i}{a}+1, \frac{p}{c}(n+j-m+1)-\frac{i}{a}\right) .
\end{aligned}
$$

The Mean of the $X_{m: n}$ is given by

$$
E\left(X_{m: n}\right)=\frac{\sum_{j=0}^{m-1}(-1)^{j}\left(\begin{array}{l}
m-1 \\
j
\end{array}\right)}{B(m, n-m+1)} p \sum_{i=0}^{\infty} \frac{c^{-\frac{(a+i)}{a}}}{i !} B\left(\frac{i}{a}+1, \frac{p}{c}(n+j-m+1)-\frac{i}{a}\right) .
$$




\subsection{L-Moments and TL-Moments}

$L$-moments (Hosking, 1990) are used to estimate the parameters. The $r^{\text {th }} L$-moment for a MLBXII distribution is given by

$$
\begin{aligned}
\lambda_{r+1} & =\frac{1}{r+1} \sum_{k=0}^{r}(-1)^{k}\left(\begin{array}{l}
r \\
k
\end{array}\right) E\left(X_{r+1-k: r+1}\right), \quad r=0,1,2,3, \ldots \\
& =\frac{1}{r+1} \sum_{k=0}^{r}(-1)^{k}\left(\begin{array}{l}
r \\
k
\end{array}\right) \int x f_{r+1-k: r+1}(x) d x \\
& =\sum_{k=0}^{r}(-1)^{k}\left(\begin{array}{l}
r \\
k
\end{array}\right)^{2} \int x[F(x)]^{r-k}[1-F(x)]^{k} f(x) d x .
\end{aligned}
$$

The Legendre polynomial is

$$
P_{r}(F(x))=\sum_{k=0}^{r}(-1)^{k}\left(\begin{array}{l}
r \\
k
\end{array}\right)^{2}[F(x)]^{r-k}[1-F(x)]^{k},
$$

and the $r^{\text {th }}$ shifted polynomial is

$$
P_{r}^{*}(F(x))=P_{r}(2 F(x)-1)=\sum_{k=0}^{r}(-1)^{r-k}\left(\begin{array}{l}
r \\
k
\end{array}\right)\left(\begin{array}{c}
r+k \\
k
\end{array}\right)[F(x)]^{k} .
$$

According to Hosking (1990), the $r^{\text {th }} L$-moment for a probability distribution is

$$
\lambda_{r+1}=\sum_{k=0}^{r}(-1)^{r-k}\left(\begin{array}{l}
r \\
k
\end{array}\right)\left(\begin{array}{c}
r+k \\
k
\end{array}\right) \int x[F(x)]^{k} f(x) d x .
$$

The $r^{\text {th }} L$-moment of a MLBXII distribution is given by

$$
\lambda_{r+1}=\sum_{k=0}^{r}(-1)^{r-k}\left(\begin{array}{l}
r \\
k
\end{array}\right)\left(\begin{array}{c}
r+k \\
k
\end{array}\right) \sum_{j=0}^{k}(-1)^{j}\left(\begin{array}{l}
k \\
j
\end{array}\right) p \sum_{i=0}^{\infty} \frac{c^{-\frac{(a+i)}{a}}}{i !} B\left(\frac{i}{a}+1, \frac{p}{c}(j+1)-\frac{i}{a}\right) .
$$

The probability weighted moments (PWM) for MLBXII distribution are

$$
l_{k}=\sum_{j=0}^{k}(-1)^{j}\left(\begin{array}{l}
k \\
j
\end{array}\right) p \sum_{i=0}^{\infty} \frac{c^{-\frac{(a+i)}{a}}}{i !} B\left(\frac{i}{a}+1, \frac{p}{c}(j+1)-\frac{i}{a}\right), \quad k=0,1,2,3, \ldots
$$


TL-moments (Elamir and Seheult, 2003) are stronger than L-moments due to trimming of outliers. TL-moments provide best estimates of the parameters for the probability distributions. The $r^{\text {th }} T L$-moment for a probability distribution is defined as

$$
\lambda_{r}^{(1)}=\frac{1}{r} \sum_{k=0}^{r-1}(-1)^{k} C_{k}^{r-1} E\left(X_{r+k+1: r+2}\right)
$$

where $E\left(X_{r+k+1: r+2}\right)=(r+k+1)\left(\begin{array}{c}r+k+1 \\ r+2\end{array}\right) \int x[F(x)]^{r+k+1}[1-F(x)]^{k-1} d F(x)$.

The $r^{\text {th }}$ TL-moment for a MLBXII distribution is given by

$$
\begin{aligned}
\lambda_{r}^{(1)}=\frac{1}{r} \sum_{k=0}^{r-1}(-1)^{k} C_{k}^{r-1}\left\{(r+k+1)\left(\begin{array}{c}
r+k+1 \\
r+2
\end{array}\right) \sum_{l=0}^{r+k+1}(-1)^{l}\left(\begin{array}{c}
r+k+1 \\
l
\end{array}\right) p\right. \\
\left.\times \sum_{i=0}^{\infty} \frac{c^{-\frac{(a+i)}{a}}}{i !} B\left(\frac{i}{a}+1, \frac{p}{c}(r+2 k+1-l)-\frac{i}{a}\right)\right\} .
\end{aligned}
$$

\subsection{Incomplete Moments}

Incomplete moments are used to study the mean inactivity life, the mean residual life function and other inequality measures. The lowest incomplete moments of the random variable $X$ with MLBXII distribution are

$$
M_{r}^{\prime}(z)=E_{X \leq z}\left(X^{r}\right)=\int_{1}^{z} x^{r} \frac{a p}{x}(\ln x)^{a-1}\left(1+c(\ln x)^{a}\right)^{-\left(\frac{p}{c}+1\right)} d x .
$$

Let $c(\ln x)^{a}=y$. Then, we have

$$
\begin{aligned}
M_{r}^{\prime}(z) & =p \int_{0}^{c(\ln z)^{a}}\left(e^{\left.y^{\frac{1}{a}}\right)^{r}}(1+y)^{-\left(\frac{p}{c}+1\right)} d y\right. \\
& =p \sum_{i=0}^{\infty} \frac{r^{i}}{i !} \int_{0}^{c(\ln z)^{a}} y^{\frac{i}{a}}(1+y)^{-\left(\frac{p}{c}+1\right)} d y \\
& =E_{X \leq z}\left(X^{r}\right)=p \sum_{i=0}^{\infty} \frac{r^{i}}{i !} d_{i}(z, a, c, p),
\end{aligned}
$$

where $d_{i}(z ; a, c, p)=c^{-\frac{(a+i)}{a}} B_{c(\ln z)^{a}}\left(\frac{i}{a}+1, \frac{p}{c}-\frac{i}{a}\right)$ and $B_{c(\ln z)^{a}}(\ldots)$ is the incomplete beta function. 
The upper incomplete moments for the random variable $X$ with a MLBXII distribution are

$$
\begin{aligned}
E_{X>z}\left(X^{r}\right) & =\int_{z}^{\infty} x^{r} f(x) d x \\
& =\int_{1}^{\infty} x^{r} f(x) d x-\int_{1}^{z} x^{r} f(x) d x \\
& =\mu_{r}^{\prime}-M_{r}^{\prime}(z) .
\end{aligned}
$$

From (4.1) and (4.23), we have

$$
E_{X>z}\left(X^{r}\right)=p \sum_{i=1}^{\infty} \frac{r^{i}}{i !}\left[d_{i}(a, c, p)-d_{i}(z ; a, c, p)\right] .
$$

The mean deviation about the mean is $M D_{\bar{X}}=E\left|X-\mu_{1}^{1}\right|=2 \mu_{1}^{1} F\left(\mu_{1}^{1}\right)-2 \mu_{1}^{1} M_{1}^{\prime}\left(\mu_{1}^{1}\right)$ and the mean deviation about the median is $M D_{M}=E|X-M|=2 M F(M)-2 M M_{1}^{\prime}(M)$, where $\mu_{1}^{\prime}=E(X)$ and $M=Q(0.5)$. Bonferroni and Lorenz curves for a specified probability $p$ are computed by $B(p)=M_{1}^{\prime}(q) / p \mu_{1}^{1}$ and $L(p)=M_{1}^{\prime}(q) / \mu_{1}^{1}$, where $q=Q(p)$.

\subsection{Residual Life functions}

The residual life, say, $m_{n}(z)$ of $X$ with a MLBXII distribution has following $n^{\text {th }}$ moment as

$$
\begin{aligned}
m_{n}(z) & =E\left[(X-z)^{n} \mid X>z\right] \\
& =(1-F(z))^{-1} \int_{z}^{\infty}(x-z)^{s} f(x) d x \\
& =(1-F(z))^{-1} \sum_{s=0}^{n}\left(\begin{array}{l}
n \\
s
\end{array}\right)(-z)^{n-s} E_{X>z}\left(X^{s}\right) .
\end{aligned}
$$

Using (4.24), we reach at

$$
m_{n}(z)=(1-F(z))^{-1} \sum_{s=0}^{n}\left(\begin{array}{l}
n \\
s
\end{array}\right)(-z)^{n-s} p \sum_{i=1}^{\infty} \frac{s^{i}}{i !}\left[d_{i}(a, c, p)-d_{i}(z ; a, c, p)\right] .
$$

The average remaining lifetime of a component at time $z$, say, $m_{1}(z)$ or the life expectancy, known as the mean residual life (MRL) function, is given by

$$
m_{1}(z)=(1-F(z))^{-1} \sum_{s=0}^{1}\left(\begin{array}{l}
1 \\
s
\end{array}\right)(-z)^{1-s} p \sum_{i=1}^{\infty} \frac{s^{i}}{i !}\left[d_{i}(a, c, p)-d_{i}(z ; a, c, p)\right] .
$$


The reverse residual life, say, $M_{n}(z)$ of $X$ with a MLBXII distribution having $n^{\text {th }}$ moment is

$$
\begin{aligned}
M_{n}(z) & =E\left[(z-X)^{n} \mid X \leq z\right] \\
& =\frac{1}{F(z)} \int_{1}^{z}(z-x)^{n} f(x) d x \\
& =\frac{1}{F(z)} \sum_{s=0}^{n}(-1)^{s}\left(\begin{array}{l}
n \\
s
\end{array}\right) z^{n-s} E_{X \leq z}\left(X^{s}\right) .
\end{aligned}
$$

Using (4.23), we reach at

$$
M_{n}(z)=\frac{1}{F(z)} \sum_{s=0}^{n}(-1)^{s}\left(\begin{array}{l}
n \\
s
\end{array}\right) z^{n-s} p \sum_{i=0}^{\infty} \frac{s^{i}}{i !} d_{i}(z ; a, c, p) .
$$

The waiting time $z$ for the failure of a component has passed with the condition that this failure had happened in the interval $[0, z]$ which is called the mean waiting time (MWT) or mean inactivity time. The waiting time $z$ for the failure of a component of $X$ having a MLBXII distribution is defined by

$$
M_{1}(z)=\frac{1}{F(z)} \sum_{s=0}^{1}(-1)^{s}\left(\begin{array}{l}
1 \\
s
\end{array}\right) z^{1-s} p \sum_{i=0}^{\infty} \frac{s^{i}}{i !} d_{i}(z ; a, c, p)
$$

Kandil et al. (2010) developed the median inactivity time function. The median inactivity time function in terms of the cdf of a continuous lifetime distribution is

$$
\operatorname{MDIT}(z)=z-F_{X}^{-1}\left(\frac{1}{2} F_{X}(z)\right)
$$

The median inactivity time function in terms of cdf of a MLBXII distribution is written as

$$
\operatorname{MDIT}(z)=z-\exp \left(c^{-\frac{1}{a}}\left[\left(1-\frac{1}{2}\left(1-\left[1+c(\ln z)^{a}\right]^{-\frac{p}{c}}\right)\right)^{-\frac{c}{p}}-1\right]^{\frac{1}{a}}\right) .
$$

\section{Reliability and Uncertainty Measures}

In this section, reliability and uncertainty measures are studied. 


\subsection{Stress-Strength Reliability for MLBXII Distribution}

If $X_{1} \sim \operatorname{MLBXII}\left(a, c, p_{1}\right), X_{2} \sim \operatorname{MLBXII}\left(a, c, p_{2}\right)$ such that $X_{1}$ represents "strength" and $X_{2}$ represents "stress" and $X_{1}, X_{2}$ follow a joint pdf $f\left(x_{1}, x_{2}\right)$, then, $R=\operatorname{Pr}\left(X_{2}<X_{1}\right)$ is the

reliability parameter. Thus, $R=\operatorname{Pr}\left(X_{2}<X_{1}\right)=\int_{1}^{\infty} f_{x_{1}}(x) F_{x_{2}}(x) d x$ is the characteristic of the distribution of $X_{1}$ and $X_{2}$, then, the reliability of the component is computed using pdf (2.5) and cdf (2.6) of MLBXII distribution as

$$
\begin{aligned}
R & =\int_{1}^{\infty} f_{x_{1}}(x) F_{x_{2}}(x) d x \\
& =\int_{1}^{\infty} \frac{a p_{1}}{x}(\ln x)^{a-1}\left(1+c(\ln x)^{a}\right)^{-\left(\frac{p_{1}}{c}+1\right)}\left[1-\left(1+c(\ln x)^{a}\right)^{-\frac{p_{2}}{c}}\right] d x \\
& =1-\int_{1}^{\infty} \frac{a p_{1}}{x}(\ln x)^{a-1}\left(1+c(\ln x)^{a}\right)^{-\left(\frac{p_{1}}{c}+\frac{p_{2}}{c}+1\right)} d x \\
& =\frac{p_{2}}{\left(p_{1}+p_{2}\right)}
\end{aligned}
$$

Therefore, $R$ is independent of $a$ and $c$. For $p_{1}=p_{2}, R=0.5$, it means that $X_{1}$ and $X_{2}$ are independently and identically distributed (i.i.d) and there is equal chance that $X_{1}$ is bigger than $X_{2}$.

\subsection{Estimation of Multicomponent Stress-Strength System Reliability for MLBXII Distribution}

Suppose a machine has at least $s$ components working out of $k$ components. The strengths of all components of the system are $X_{1}, X_{2}, \ldots, X_{k}$ and the stress $Y$ is applied to the system. Both strengths $X_{1}, X_{2}, \ldots, X_{k}$ and the stress $Y$ are i.i.d. random variables. $G$ is the cdf of $Y$ and $F$ is the cdf of $X$. The reliability of a machine is the probability that the machine functions properly, i.e.,

$$
\begin{aligned}
R_{s, k} & =P(\text { strengths }>\text { stress }) \\
& =P\left[\text { at least "s" of }\left(X_{1}, X_{2}, \ldots . X_{k}\right) \text { exceed } Y\right]
\end{aligned}
$$

If $X \sim \operatorname{MLBXII}\left(a, c, p_{1}\right)$ and $Y \sim \operatorname{MLBXII}\left(a, c, p_{2}\right)$ with unknown shape parameters $p_{1}, p_{2}$ and common scale parameter $b$, where $X$ and $Y$ are independently distributed, 
the reliability in the multicomponent stress - strength for a MLBXII distribution is as

$$
\begin{aligned}
R_{s, k} & =\sum_{i=s}^{k}\left(\begin{array}{l}
k \\
i
\end{array}\right) \int_{-\infty}^{\infty}[1-F(y)]^{i}[F(y)]^{k-i} d G(y) \text { (Bhattacharyya and Johnson , 1974) } \\
& =\sum_{i=s}^{k}\left(\begin{array}{l}
k \\
i
\end{array}\right) \int_{1}^{\infty}\left(\left[1+c(\ln y)^{a}\right]^{-\frac{p_{1}}{c}}\right)^{i}\left(1-\left[1+c(\ln y)^{a}\right]^{-\frac{p_{1}}{c}}\right)^{(k-i)} \frac{a p_{2}}{y} \\
& \times(\ln y)^{a-1}\left[1+c(\ln y)^{a}\right]^{-\left(\frac{p_{2}}{c}+1\right)} d y
\end{aligned}
$$

Let $t=\left[1+c(\ln y)^{a}\right]^{-\frac{p_{2}}{c}}$, then we obtain

$$
R_{s, k}=\sum_{i=s}^{k}\left(\begin{array}{l}
k \\
i
\end{array}\right) \int_{0}^{1}\left(t^{v}\right)^{i}\left(1-t^{v}\right)^{(k-i)} d t
$$

Let $z=t^{v}, t=z^{\frac{1}{v}}$, and $t=\frac{1}{v} z^{\frac{1}{v}-1} d z$. Then,

$$
\begin{aligned}
R_{s, k} & =\sum_{i=s}^{k}\left(\begin{array}{l}
k \\
i
\end{array}\right) \int_{0}^{1}(z)^{i}(1-z)^{(k-i)} \frac{1}{v} z^{\frac{1}{v}-1} d z \\
& =\frac{1}{v} \sum_{i=s}^{k}\left(\begin{array}{l}
k \\
i
\end{array}\right) \int_{0}^{1}(z)^{i+\frac{1}{v}-1}(1-z)^{(k-i)} d z \\
& =\frac{1}{v} \sum_{i=s}^{k}\left(\begin{array}{l}
k \\
i
\end{array}\right) B\left(i+\frac{1}{v}, k-i+1\right),
\end{aligned}
$$

where $v=\frac{p_{1}}{p_{2}}$

$$
R_{s, k}=\frac{1}{v} \sum_{i=s}^{k}\left(\begin{array}{l}
k \\
i
\end{array}\right) B\left(i+\frac{1}{v}, k-i+1\right)=\frac{1}{v} \sum_{i=s}^{k} \frac{k !}{(k-i) !}\left(\prod_{j=0}^{i}(k+v-j)\right)^{-1} .
$$

The probability $R_{s, k}$ in (5.5) is called the multicomponent stress-strength model reliability.

\subsection{Shannon Entropy and Awad Entropy}

According to Shannon (1948), the measurement of the expected information in a message is called entropy. Shannon entropy for a random variable $X$ with the pdf (2.5) 
is given by

$$
\begin{aligned}
h(X) & =E(I(X))=\int \ln \left(\frac{1}{f(x)}\right) f(x) d x \\
& =-\int_{1}^{\infty} \ln \left\{\frac{a p}{x}(\ln x)^{a-1}\left[1+c(\ln x)^{a}\right]^{-\left(\frac{p}{c}+1\right)}\right\}\left(\frac{a p}{x}(\ln x)^{a-1}\left[1+c(\ln x)^{a}\right]^{-\left(\frac{p}{c}+1\right)}\right) d x \\
& =\left[p d_{1}(a, c, p)-\ln (a p)+\frac{(a-1)}{a}\left[\psi\left(\frac{p}{c}\right)-\gamma-\ln c\right]-\left(\frac{c}{p}+1\right)\right]
\end{aligned}
$$

where $\psi\left(\frac{\mathrm{p}}{c}\right)$ is the digamma function and $\psi(1)=\gamma$.

Awad (1987) provided the extension of Shannon entropy as

$$
A(X)=-\int_{0}^{\infty} f(x) \ln \frac{f(x)}{\delta} d x,
$$

where $\delta$ is the maximum value of the ordinate of a MLBXII distribution in the domain of $X$.

If a random variable $X$ has a MLBXII distribution, then Awad entropy is given by

$$
A(X)=\left[\ln \delta+p d_{1}(a, c, p)-\ln (a p)+\frac{(a-1)}{a}\left[\psi\left(\frac{\mathrm{p}}{c}\right)-\gamma-\ln c\right]-\left(\frac{c}{p}+1\right)\right] .
$$

\subsection{Renyi Entropy, Q-Entropy, Havrda and Chavrat Entropy and Tsallis- Entropy}

Renyi entropy is an extension of Shannon entropy. Renyi entropy for a MLBXII random variable $X$ with the pdf (2.5) is theoretically computed as

$$
\begin{aligned}
I_{R} & =\frac{1}{1-v} \log \left\{\int_{1}^{\infty}[f(x)]^{v}\right\} d x, \quad v \neq 1, \quad v>0, \\
& =\frac{1}{1-v} \log \left\{\int_{1}^{\infty}\left[\frac{a p}{x}(\ln x)^{a-1}\left[1+c(\ln x)^{a}\right]^{-\left(\frac{p}{c}+1\right)}\right]^{v}\right\} d x, \quad v \neq 1, \quad v>0 .
\end{aligned}
$$

In addition,

$$
\int_{1}^{\infty}\left[\frac{a p}{x}(\ln x)^{a-1}\left[1+c(\ln x)^{a}\right]^{-\left(\frac{p}{c}+1\right)}\right]^{v} d x=\frac{a^{v} p^{v}}{\Gamma\left[v\left(\frac{p}{c}+1\right)\right]} \sum_{k=0}^{\infty} \frac{(-1)^{k} \Gamma\left(v\left(\frac{p}{c}+1\right)+k\right) c^{k}}{k !} \frac{\Gamma(a(v+k)-v+1)}{(v-1)^{(a(v+k)-v+1)}} .
$$


Therefore,

$$
I_{R}=\frac{1}{1-v} \log \left\{\frac{a^{v} p^{v}}{\Gamma\left[v\left(\frac{p}{c}+1\right)\right]} \sum_{k=0}^{\infty} \frac{(-1)^{k} \Gamma\left(v\left(\frac{p}{c}+1\right)+k\right) c^{k}}{k !} \frac{\Gamma(a(v+k)-v+1)}{(v-1)^{(a(v+k)-v+1)}}\right\}
$$

The Q-entropy for a MLBXII distribution is

$$
\begin{aligned}
H_{q}(f(x)) & =\frac{1}{1-q} \log \left\{1-\int_{1}^{\infty}[f(x)]^{q} d x\right\}, \quad q \neq 1, q>0, \\
& =\frac{1}{1-q} \log \left[1-\frac{a^{q} p^{q}}{\Gamma\left[q\left(\frac{p}{c}+1\right)\right]} \sum_{k=0}^{\infty} \frac{(-1)^{k} \Gamma\left(q\left(\frac{p}{c}+1\right)+k\right) c^{k}}{k !} \frac{\Gamma(a(q+k)-q+1)}{(q-1)^{(a(q+k)-q+1)}}\right] .
\end{aligned}
$$

The Havrda and Chavrat entropy for a MLBXII distribution is

$$
\begin{aligned}
S_{H C}(f(x)) & =\frac{1}{v-1} \log \left\{\int_{1}^{\infty}[f(x)]^{v} d x\right\}, \quad v \neq 1, v>0, \\
& =\frac{1}{v-1} \log \left\{\frac{a^{v} p^{v}}{\Gamma\left[v\left(\frac{p}{c}+1\right)\right]} \sum_{k=0}^{\infty} \frac{(-1)^{k} \Gamma\left(v\left(\frac{p}{c}+1\right)+k\right) c^{k}}{k !} \frac{\Gamma(a(v+k)-v+1)}{(v-1)^{(a(v+k)-v+1)}}\right\} .
\end{aligned}
$$

The Tsallis-entropy for a MLBXII distribution is

$$
\begin{aligned}
S_{q}(f(x)) & =\frac{1}{q-1} \log \left\{1-\int_{1}^{\infty}[f(x)]^{q} d x\right\}, \quad q \neq 1, q>0, \\
& =\frac{1}{q-1}\left(1-\frac{a^{q} p^{q}}{\Gamma\left[q\left(\frac{p}{c}+1\right)\right]} \sum_{k=0}^{\infty} \frac{(-1)^{k} \Gamma\left(q\left(\frac{p}{c}+1\right)+k\right) c^{k}}{k !} \frac{\Gamma(a(q+k)-q+1)}{(q-1)^{(a(q+k)-q+1)}}\right) .
\end{aligned}
$$

Shannon entropy, Collision entropy, Hartley entropy and Min entropy can be obtained from Renyi entropy. For $v \rightarrow 1$, Renyi entropy inclines to Shannon entropy. For $v \rightarrow 2$, Renyi entropy inclines to quadratic entropy. Entropies are applied to study heart beat intervals, Cardiac Autonomic Neuropathy (CAN), DNA sequences, anomalous diffusion, daily temperature fluctuations (climatic), and information content signals. 


\section{Compounding and Probability Distribution}

In this section, a MLBXII distribution is obtained from some compound scale mixture of GLW, gamma and size-biased Erlang distributions.

\subsection{Development of Log-Weibull Distribution}

If $c \rightarrow 0$, a MLBXII distribution will reduce to the log Weibull distribution with the pdf

$$
w(x, a)=\frac{a}{x}(\ln x)^{a-1} e^{-(\ln x)^{a}}, \quad x>1, a>0 .
$$

and the cdf

$$
W(x, a)=1-e^{-(\ln x)^{a}}, \quad x \geq 1, a>0 .
$$

\subsection{Compound Scale Mixture of GLW Distribution with Gamma Distribu- tion}

Theorem 6.1. Let $w(x, a, c / \theta)=\frac{a c \theta}{x}(\ln x)^{a-1} e^{-\theta c(\ln x)^{a}}, x>1$ be the pdf of GLW distribution and let $\theta$ have a gamma distribution with the pdf $g(\theta, \alpha, p, c)=\frac{\alpha^{\frac{p}{c}}}{\Gamma\left(\frac{p}{c}\right)} \theta^{\frac{p}{c}-1} e^{-\alpha \theta}, \theta>0$. Then $X$ has the $p d f(2.5)$.

Proof. For compounding $f(x, a, c, p)=\int_{0}^{\infty} w(x, a, c / \theta) g(\theta, \alpha, c, p) d \theta$,

$$
\begin{aligned}
f(x, a, c, p) & =\int_{0}^{\infty} \frac{a c \theta}{x}(\ln x)^{a-1} e^{-\theta c(\ln x)^{a}} \frac{1}{\Gamma\left(\frac{p}{c}\right)} \theta^{\frac{p}{c}-1} e^{-\theta} d \theta \\
& =\frac{a p}{x}(\ln x)^{a-1}\left[1+c(\ln x)^{a}\right]^{-\left(\frac{p}{c}+1\right)}, \quad x>1 .
\end{aligned}
$$

is the pdf of a MLBXII distribution.

\subsection{Compound Scale Mixture of GLW Distribution and Size-biased Erlang Distribution.}

Theorem 6.2. Let $w(x, a, c, \alpha / \theta)=\frac{a c \theta}{x}(\ln x)^{a-1} e^{-\theta c(\ln x)^{a}}, x>1$ be the pdf of a GLW distribution and $\theta$ has a size-biased moment Erlang distribution with the $p d f$

$$
g(\theta, \gamma, c, p)=\frac{\alpha^{\frac{p}{c}-\gamma}}{\Gamma\left(\frac{p}{c}-\gamma\right)} \theta^{\frac{p}{c}-\gamma-1} e^{-\alpha \theta}, \quad \theta>0 .
$$

Then, X has the pdf (2.5). 
Proof. For compounding $f(x, a, c, p)=\int_{0}^{\infty} w(x, a, c / \theta) g(\theta, \alpha, c, p) d \theta$,

$$
\begin{aligned}
f(x, a, c, p) & =\int_{0}^{\infty} \frac{a c \theta}{x}(\ln x)^{a-1} e^{-\theta c(\ln x)^{a}} \frac{\alpha^{\frac{p}{c}-\gamma}}{\Gamma\left(\frac{p}{c}-\gamma\right)} \theta^{\frac{p}{c}-\gamma-1} e^{-\alpha \theta} d \theta \\
& =\frac{a c\left(\frac{p}{c}-\gamma\right)}{x}(\ln x)^{a-1}\left[1+c(\ln x)^{a}\right]^{-\left(\frac{p}{c}-\gamma+1\right)} .
\end{aligned}
$$

For $\gamma=0$, we have that $f(x, a, c, p)=\frac{a p}{x}(\ln x)^{a-1}\left[1+c(\ln x)^{a}\right]^{-\left(\frac{p}{c}+1\right)}$ is the pdf of a MLBXII distribution.

\section{Characterizations}

In this section, a MLBXII distribution is characterized through (i) truncated moment of the log of random variable (ii) the truncated moment of a function (not log) of a random variable, (iii) the hazard function, (iv) Mills ratio, (v) certain functions of the random variable, (vi) the distribution of the $1^{\text {st }}$ order statistic, and (vii) the conditional expectation of record values. One of the advantages of the characterization (ii) is that the cdf is not required to have a closed form. We present our characterizations (i) - (vii) in seven subsections.

\subsection{Characterization Based on Truncated Moment of Log of the Random Variable}

In this subsection, the first characterization of a MLBXII distribution is discussed.

Proposition 7.1. Let $X: \Omega \rightarrow(1, \infty)$ be a continuous random variable with the cdf $F(x)$, $(0<F(x)<1$ for $x>1)$. Then, for $p>c, X$ has the $c d f$ (2.6) if and only if

$$
E\left[(\ln X)^{a} \mid X>t\right]=\frac{1}{p-c}\left\{p(\ln (t))^{a}+1\right\}, \quad \text { for } \quad t>1 .
$$

Proof. If $\mathrm{X}$ has the cdf (2.6), then

$$
\begin{aligned}
E\left[(\ln (X))^{a} \mid X>t\right] & =(1-F(t))^{-1} \int_{t}^{\infty}(\ln x)^{a} f(x) d x \\
& =\left[1+c(\ln t)^{a}\right]^{\frac{p}{c}} \int_{t}^{\infty}(\ln x)^{a} \times \frac{a p}{x}(\ln x)^{a-1}\left[1+c(\ln x)^{a}\right]^{-\left(\frac{p}{c}+1\right)} d x .
\end{aligned}
$$


Upon integration by parts and simplification, we obtain

$$
E\left[(\ln X)^{a} \mid X>t\right]=\frac{1}{p-c}\left\{p(\ln (t))^{a}+1\right\}, \quad \text { for } \quad t>1 .
$$

Conversely, if (7.1) holds, then

$$
\int_{t}^{\infty}(\ln X)^{a} f(x) d x=\frac{1}{p-c}\left\{p(\ln (t))^{a}+1\right\} .
$$

Therefore,

$$
(1-F(t))^{-1} \int_{t}^{\infty}(\ln x)^{a} f(x) d x=\frac{(1-F(t))}{p-c}\left\{p(\ln (t))^{a}+1\right\} .
$$

Differentiating both sides of the above equation with respect to $t$ we obtain

$$
-(\ln t)^{2 a} f(t)=\frac{(1-F(t))}{p-c}\left(\frac{a p}{t}(\ln (t))^{a-1}\right)-\frac{f(t)}{p-c}\left(p(\ln (t))^{a}+1\right) .
$$

After simplification and integration we obtain

$$
F(t)=1-\left[1+c(\ln t)^{a}\right]^{-\frac{p}{c}}, \quad \text { for } \quad t \geq 1 .
$$

\subsection{Characterizations Based on Truncated Moments of a Function of the Random Variable}

In this subsection, we first present a characterization of a MLBXII distribution in terms of a simple relationship between the truncated moment of a function of $X$ and another function. This characterization result employs a version of the theorem due to Glänzel (1987); see Theorem 9.1 of Appendix A. Note that the result holds also when the interval $H$ is not closed. Moreover, as mentioned above, it could be also applied, when the cdf $F$ does not have a closed form. As shown in Glänzel (1990), this characterization is stable in the sense of a weak convergence.

Proposition 7.2. Let $X: \Omega \rightarrow(1, \infty)$ be a continuous random variable and let

$$
q(x)=\left[1+c(\ln x)^{a}\right]^{-1}, \quad \text { for } \quad x>1 .
$$

The random variable $X$ has a $p d f(2.5)$ if and only if the function $\eta$ defined in Theorem 9.1 has the form $\eta(x)=\frac{p}{p+c}\left[1+c(\ln x)^{a}\right]^{-1}$, for $x>1$. 
Proof. If $X$ has the pdf (2.5), then

$$
(1-F(x)) E(q(X) \mid X \geq x)=\frac{p}{p+c}\left[1+c(\ln x)^{a}\right]^{-\left(\frac{p}{c}+1\right)}, \quad x>1 .
$$

or

$$
E(q(X) \mid X \geq x)=\frac{p}{p+c}\left[1+c(\ln x)^{a}\right]^{-1}, \quad x>1 .
$$

Thus, $\eta(x)=\frac{p}{p+c}\left[1+c(\ln x)^{a}\right]^{-1}, x>1$ and

$$
\eta(x)-q(x)=-\frac{c}{p+c}\left[1+c(\ln x)^{a}\right]^{-1}<0, \quad \text { for } \quad x \geq 1 .
$$

Conversely, if $\eta$ is given as above, then

$$
s^{\prime}(x)=\frac{\eta^{\prime}(x)}{\eta(x)-q(x)}=\frac{\frac{a p}{x}(\ln x)^{a-1}}{\left[1+c(\ln x)^{a}\right]^{\prime}}, \quad x>1,
$$

and hence

$$
s(x)=\ln \left\{\left[1+c(\ln x)^{a}\right]^{\frac{p}{c}}\right\}, \quad x>1,
$$

and

$$
e^{-s(x)}=\left[1+(\ln x)^{a}\right]^{-\frac{p}{c}}, \quad x>1 .
$$

In view of Theorem 9.1, X has the density (2.5).

Corollary 7.1. Let $X: \Omega \rightarrow(1, \infty)$ be a continuous random variable. The pdf of $X$ is $(2.5)$ if and only if there exists functions $\eta(x)$ and $q(x)$ defined in Theorem 9.1 satisfying the differential equation

$$
\frac{\eta^{\prime}(x)}{\eta(x)-q(x)}=\frac{\frac{a p}{x}(\ln x)^{a-1}}{\left[1+c(\ln x)^{a}\right]} .
$$

The general solution of the differential equation in Corollary 7.1 is

$$
\eta(x)=\left[1+c(\ln x)^{a}\right]^{\frac{p}{c}}\left[-\int \frac{\frac{a p}{x}(\ln x)^{a-1}}{\left[1+c(\ln x)^{a}\right]^{\left(\frac{p}{c}+1\right)}} q(x) d x\right]+D
$$

where $D$ is a constant. Note that a set of functions satisfying above differential equation is given in Proposition 7.2 with $D=0$. However, it should also be noted that there are other pairs $(\eta, q)$ satisfying the conditions of Theorem 6.1 . 


\subsection{Characterization Based on Hazard Function}

It is well-known that the hazard function $h_{F}$ of a twice differential distribution function $F$ satisfies the first order differential equation

$$
\frac{d}{d x}[\log f(x)]=\frac{h_{F}^{\prime}(x)}{h_{F}(x)}-h_{F}(x) .
$$

For many univariate continuous distributions, this is the only characterization available in terms of the hazard function. The following characterization establishes a characterization of a MLBXII distribution which is not of the above trivial form.

Proposition 7.3. Let $X: \Omega \rightarrow(1, \infty)$ be a continuous random variable. The $p d f$ of $X$ is $(2.5)$, if and only if its hazard function $h_{F}$ satisfies the first order differential equation

$$
\left(x h_{F}^{\prime}+h_{F}\right)=\frac{a p(\ln x)^{a-2}\left[(a-1)-c(\ln x)^{a}\right]}{x\left[1+c(\ln x)^{a}\right]^{2}} .
$$

Proof. If $X$ has the pdf (2.5), then the above differential equation holds. Now, if the differential equation holds, then

$$
\frac{d}{d x}\left\{x h_{F}(x)\right\}=\operatorname{ap} \frac{d}{d x}\left[\frac{(\ln x)^{a-1}}{\left[1+c(\ln x)^{a}\right]}\right] .
$$

or

$$
h_{F}(x)=\frac{a p(\ln x)^{a-1}}{x\left[1+c(\ln x)^{a}\right]}, \quad x>1 .
$$

which is the hazard function of a MLBXII distribution.

\subsection{Characterization through Mills Ratio}

In this subsection, we introduce the characterization of a MLBXII distribution via Mills ratios.

Definition 7.1. Let $X: \Omega \rightarrow(1, \infty)$ be a continuous random variable. The function $f(x)$ is the pdf of $X$, if and only if the Mills ratio $m(x)$ is twice differentiable and satisfying the equation

$$
\frac{d}{d x}[\ln f(x)]+\frac{\left(m^{\prime}(x)+1\right)}{m(x)}=0
$$


Proposition 7.4. Let $X: \Omega \rightarrow(1, \infty)$ be a continuous random variable. The function $f(x)$ is the pdf of $X$, if and only if the Mills ratio $m(x)$ is twice differentiable and satisfying the equation

$$
m_{F}^{\prime}(x)-x^{-1} m(x)=\frac{x}{a p}\left[c-(a-1)(\ln x)^{-a}\right] .
$$

Proof. If (6) is the pdf of $X$, then (7.2) holds. Now, if (7.2) holds, then

$$
\begin{aligned}
& \frac{d}{d x}\left(x^{-1} m(x)\right)=\frac{1}{a p} \frac{d}{d x}\left(\left[(\ln x)^{-a+1}+c(\ln x)\right]\right), \\
& m(x)=\frac{1-F(x)}{f(x)}=\frac{x}{a p}(\ln x)^{-a+1}\left[1+c(\ln x)^{a}\right], \quad x>1 .
\end{aligned}
$$

which is the Mills ratio of the MLBXII distribution.

\subsection{Characterization Based on Certain Functions of the Random Variable}

The following proposition has already appeared in Hamedani (2013). So we will just state it here which can be used to characterize a MLBXII distribution.

Proposition 7.5. Let $X: \Omega \rightarrow(1, \infty)$ be a continuous random variable with the cdf $(2.6)$. Let $\psi(x)$ be a differentiable function on $(1, \infty)$ with $\lim _{x \rightarrow 1+}, \psi(x)=1$. Then, for $\delta \neq 1$,

$$
E(\psi(X) \mid X>x)=\delta \psi(x), \quad x>1 \text {. }
$$

if and only if

$$
\psi(x)=(1-F(x))^{\frac{1}{\delta}-1}, \quad x>1 .
$$

Remark 1. It is easy to see that, for certain functions, e.g., $\psi(x)=\left[1+c(\ln x)^{a}\right]^{-1}$ and $\delta=\frac{p}{c+p}$, Proposition 7.5 provides a characterization of a MLBXII distribution. Clearly, there are other suitable functions $\psi(x)$, but we chose this case for the simplicity.

Proposition 7.6. Let $X: \Omega \rightarrow(1, \infty)$ be a continuous random variable with the cdf $F$ and the pdf $f$. Let $\psi(x)$ and $\varphi(x)$ be differentiable functions on $(1, \infty)$ such that $\int_{1}^{\infty} \frac{\varphi^{\prime}(x)}{\varphi(x)-\psi(x)} d x=\infty$. Then,

$$
E(\psi(X) \mid X>x)=\varphi(x), \quad x>1,
$$

implies

$$
F(x)=1-\exp \left[-\int_{1}^{x} \frac{\varphi^{\prime}(t)}{\varphi(t)-\psi(t)} d t\right], \quad x \geq 1 .
$$


Proof. We have

$$
\int_{x}^{\infty} \psi t(u) f(u) d u=(1-F(x)) \varphi(x) .
$$

Differentiating both sides of the above equation with respect to $t$ and then rearranging the terms, we arrive at

$$
\frac{f(x)}{1-F(x)}=\frac{\varphi^{\prime}(x)}{\varphi(x)-\psi(x)}, \quad x>1
$$

Integrating the last equation from 1 to $x$, we have

$$
F(x)=1-\exp \left[-\int_{1}^{x} \frac{\varphi^{\prime}(t)}{\varphi(t)-\psi(t)} d t\right], \quad x \geq 1 .
$$

Remark 2. Taking $\psi(x)=\left[1+c(\ln x)^{a}\right]^{\frac{p}{2 c}}$ and $\varphi(x)=2 \psi(x)$, Proposition 7.6 provides the characterization of (2.6). Clearly, there are other choices for these functions.

\subsection{Characterization through Distribution of the First Order Statistic}

Here we characterize the MLBXII distribution via the distribution of the first order statistic.

Proposition 7.7. Let $X_{1}, X_{2}, \ldots, X_{n}$ be independent and identically distributed random variables, if the distribution of $X_{i}^{\prime} s$ is MLBXII, then $X_{1: n}$ has a MLBXII distribution.

Proof. Observe that

$$
P_{r}\left(X_{1: n}>x\right)=\left[P_{r}\left(X_{1}>x\right)\right]^{n}=[1-F(x)]^{n}=\left[1+c(\ln x)^{a}\right]^{-\frac{n p}{c}}
$$

\subsection{Characterization via Conditional Expectation of Record Values}

Nagaraja (1988), Arnold et al. (1998), Khan and Alzaid (2004), Khan et al. (2010) and Athar et al. (2014) characterized distributions via the conditional expectation of record values. 
Proposition 7.8. Let $X: \Omega \rightarrow(1, \infty)$ be a continuous random variable with the cdf $F(x)$ and the $p d f f(x)$. Let $X_{U(r)}$ be the $r^{\text {th }}$ record values of the random sample $X_{1}, X_{2}, \ldots, X_{n}$. Then, for any two successive values $X_{U(r)}$ and $X_{U(s)}, 1 \leq r<s \leq n, E\left[\left(h\left(X_{U(s)}-X_{U(r)}\right)\right)^{q} X_{U(r)}=\right.$ $x]=a^{*} \Sigma_{j=0}^{q}\left(\begin{array}{c}q \\ j\end{array}\right)(h(x))^{q-j}\left(\frac{b}{a}\right)^{j}$ holds, if and only if $F(x)=1-[a+b h(x)]^{d}$, where $a \neq 0, a^{*}=$ $\sum_{i=0}^{q}\left(\begin{array}{l}q \\ i\end{array}\right)(-1)^{i+q}\left(\frac{d}{d+i}\right)^{s-r}$ and $h(x)$ is the differentiable function of $x$.

Remark 3. Taking $a=c, b=1, h(x)=(\ln x)^{a}, d=-\frac{p}{c}$, proposition 7.8 provides a characterization of $F(x)=1-[1+c(\ln x)]^{-\frac{p}{c}}, x \geq 1$.

\section{Maximum Likelihood Estimation}

In this section, estimates of the parameters are derived using the maximum likelihood method. The modified Log Burr XII (MLBXII), Log-Burr XII (LBXII), Log-Lomax (LL), Log-log logistic (LLL), modified Burr XII (MBXII), and Burr XII probability distributions are fitted to real data sets of strengths for single fibers for comparison purposes. Raqab and Kundu (2005), Gupta and Kundu (2010), Asgharzadeh et al. (2013) and Muhammad (2016) also studied strength measured in GPA for single fibers tested under the tension at gauge 10 millimeters. Each single fiber of the carbon was filled with 1000 carbon fiber tows. The log likelihood function for a MLBXII distribution with the parameter vector $\Phi=(a, c, p)$ is

$$
\ln L\left(x_{i}, \Phi\right)=n \ln a+n \ln p-\sum_{i=1}^{n} \ln x_{i}+(a-1) \sum_{i=1}^{n} \ln \left(\ln x_{i}\right)-\left(\frac{p}{c}+1\right) \sum_{i=1}^{n} \ln \left[1+c\left(\ln x_{i}\right)^{a}\right]
$$

In order to compute the estimates of the parameters of a MLBXII distribution, following nonlinear equations must be solved simultaneously:

$$
\begin{aligned}
& \frac{\partial \ln L}{\partial a}=\frac{n}{a}+\sum_{i=1}^{n} \ln \left(\ln x_{i}\right)-(p+c) \sum_{i=1}^{n}\left(\ln x_{i}\right)^{a} \ln \left(\ln x_{i}\right)\left[1+c\left(\ln x_{i}\right)^{a}\right]^{-1} \\
& \frac{\partial \ln L}{\partial c}=\frac{p}{c^{2}} \sum_{i=1}^{n} \ln \left[1+c\left(\ln x_{i}\right)^{a}\right]-\left(\frac{p}{c}+1\right) \sum_{i=1}^{n}\left(\ln x_{i}\right)^{a}\left[1+c\left(\ln x_{i}\right)^{a}\right]^{-1}=0 . \\
& \frac{\partial \ln L}{\partial p}=\frac{n}{p}-\frac{1}{c} \sum_{i=1}^{n} \ln \left[1+c\left(\ln x_{i}\right)^{a}\right]=0 .
\end{aligned}
$$


Table 1: MLEs and their standard errors (in parentheses) for data set.

\begin{tabular}{|c|c|c|c|}
\hline Model & $\mathrm{a}$ & $\mathrm{c}$ & $\mathrm{p}$ \\
\hline MLBXII & 7.3518694 & 0.1312964 & 0.3860578 \\
& $(1.33378429)$ & $(0.1387852)$ & $(0.08368976)$ \\
\hline LBXII & 10.6447552 & - & 0.6528913 \\
& $(1.3866202)$ & & $(0.6528913)$ \\
\hline LL & - & - & 1.357235 \\
& & & $(0.1709954)$ \\
\hline LLL & 8.51017 & - & - \\
& $(0.8752687)$ & & \\
\hline MBXII & 40.53647585 & 0.13075754 & 0.00346656 \\
& $(-)$ & $(0.0319819847)$ & $(0.0009429031)$ \\
\hline BXII & 13.27931063 & - & 0.06854902 \\
& $(47.621595)$ & & $(0.2458647)$ \\
\hline
\end{tabular}

\subsection{Application}

In order to compare a MLBXII distribution with LBXII, LL, LLL, MBXII and BXII distributions, different goodness of fit measures, like Kolmogorov Smirnov (KS), Akaike information criterion (AIC), consistent Akaike information criterion (CAIC), Bayesian information criterion (BIC), Hannan-Quinn information criterion (HQIC) and likelihood ratio statistics, are computed using an $\mathrm{R}$ package for strengths. The better fit corresponds to the smaller K-S, AIC, CAIC, BIC, HQIC and $-\ell$ values. The data of strengths are 1.901, 2.132, 2.203, 2.228, 2.257, 2.350, 2.361, 2.396, 2.397, 2.445, 2.454, $2.474,2.518,2.522,2.525,2.532,2.575,2.614,2.616,2.618,2.624,2.659,2.675,2.738,2.740$, $2.856,2.917,2.928,2.937,2.937,2.977,2.996,3.030,3.125,3.139,3.145,3.220,3.223,3.235$, $3.243,3.264,3.272,3.294,3.332,3.346,3.377,3.408,3.435,3.493,3.501,3.537,3.554,3.562$, $3.628,3.852,3.871,3.886,3.971,4.024,4.027,4.225,4.395$ and 5.020.

The maximum likelihood estimates (MLE) of parameters and values of goodness of fit measures for a MLBXII distribution, its sub-models (LBXII, LL and LLL) and competing models (MBXII and BXII) are computed. Table 1 displays MLEs and their standard errors (in parentheses) for the data set. Table 2 displays goodness of fit values.

The MLBXII distribution is best fitted compared to LBXII, LL, LLL, MBXII, BXII distributions because the values of all criteria are smaller for the MLBXII distribution. In addition, the MLBXII distribution offers closer fit to empirical data.

\section{Conclusion}

We have developed the MLBXII distribution on the basis of the generalized log Pearson differential equation (GLPE). Basic mathematical properties along with sub-models, 
Table 2: Goodness-of-fit statistics for data set.

\begin{tabular}{|c|c|c|c|c|c|c|}
\hline Model & $\mathrm{D}(\mathrm{KS})$ & AIC & CAIC & BIC & HQIC & $-\ell$ \\
\hline MLBXII & 0.0882119 & 119.8909 & 120.2977 & 126.3203 & 122.4196 & 56.94545 \\
\hline LBXII & 0.1275 & 123.0831 & 123.2831 & 127.3694 & 124.7689 & 59.54156 \\
\hline LL & 0.5188 & 320.763 & 320.8286 & 322.9061 & 321.6059 & 159.3815 \\
\hline LLL & 0.2285 & 129.1787 & 129.2443 & 131.3219 & 130.0216 & 63.58937 \\
\hline MBXII & 0.5166 & 277.2935 & 277.7003 & 283.7229 & 279.8222 & 135.6467 \\
\hline BXII & 0.4821 & 280.2543 & 284.540 & 288.9503 & 281.940 & 138.2604 \\
\hline
\end{tabular}

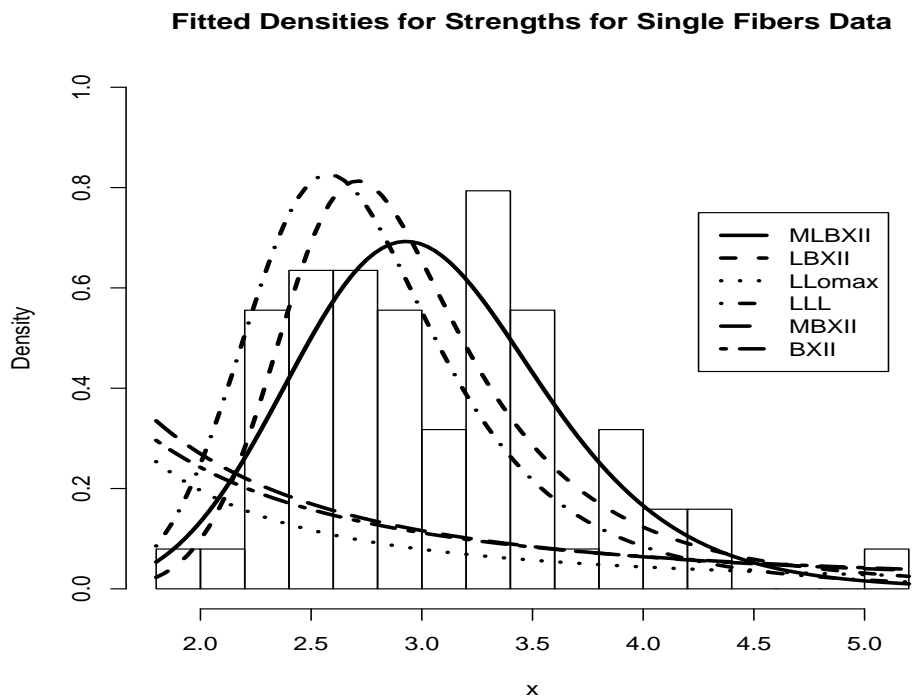

(a) Figure 3: Plots of fitted pdf's.

moments, L-moments, TL-moments, inequality measures, residual and reverse residual life functions, reliability measures, uncertainty measures, compounding and characterizations of a MLBXII distribution are taken up. Goodness of fit measures showed that MLBXII has a better fit. Application of the MLBXII model to strengths for single fibers is illustrated to show the significance and flexibility of a MLBXII distribution.

\section{Acknowledgements}

The authors are grateful to the editor, associate editor and referees for constructive comments and suggestions which improved the article greatly. 


\section{References}

Afify, A. Z., Cordeiro, G. M., Ortega, E. M., Yousof, H. M., and Butt, N. S. (2016), The Four-Parameter Burr XII Distribution: Properties, Regression Model and Applications. Communications in Statistics - Theory and Methods, (Accepted for publication)

Asgharzadeh, A., Esmaeili, L., Nadarajah, S., and Shih, S. H. (2013), A generalized skew logistic distribution. REVSTAT-Statistical Journal , 11(3), 317 - 338.

Arnold, B.C., Balakrishnan, N., and Nagarajah, H. (1998), Records, John Wiley, New York .

Athar, H., and Noor, Z. (2014), Characterization of probability distributions by conditional expectations of functions of record statistics. Journal of the Egyptian Mathematical Society, 22, 275 - 279.

Begum, A.A., and Parvin, S. (2002), Moments of order statistics from doubly truncated Burr XII distribution. Journal of Statistical Research, 36, 179 - 190.

Bhattacharyya, G. K., and Johnson, R. A. (1974), Estimation of reliability in a multicomponent stress-strength model. Journal of the American Statistical Association, 69, 966 970.

Bhatti, F. A., Ali, A., Hamedani, G.G., and Ahmad, M. (2018), On Generalized Log Burr XII Distribution. Pakistan Journal of Statistics and Operation Research, (Accepted)

Burr, I. W. (1942), Cumulative frequency functions. The Annals of Mathematical Statistics, 13, 215 - 232.

Cadena, M. (2017), Extensions of the Burr Type XII distribution and Applications. arXiv preprint arXiv:, 1705.10374.

Doǵru, F. Z., and Arslan, O. (2016), Optimal B-robust estimators for the parameters of the Burr XII distribution. Journal of Statistical Computation and Simulation, 86(6), 1133 $-1149$.

Elamir, E. A., and Seheult, A. H. (2003), Trimmed L-moments. Computational Statistics and Data Analysis, 43(3), 299 - 314.

Ghosh, I., and Bourguignon, M. (2017), A New Extended Burr XII Distribution. Austrian Journal of Statistics, 46, 33 - 39. 
Glänzel, W. A. (1987), A characterization theorem based on truncated moments and its application to some distribution families. Mathematical statistics and probability theory, $75-84$.

Glänzel, W. A. (1990), Some consequences of a characterization theorem based on truncated moments. Statistics, 21, 613 - 618.

Gomes, A. E., da-Silva, C. Q., and Cordeiro, G. M. (2015), Two extended Burr models: Theory and practice. Communications in Statistics-Theory and Methods, 44(8), 1706 1734.

Guerra, R. R., Peña - Ramírez, F. A., and Cordeiro, G. M. (2017), The gamma Burr XII distribution: Theory and application. Journal of Data Science, 15(3), 467-494.

Gupta, R.D. and Kundu, D. (2010), Generalized logistic distributions. Journal of Applied Statistical Sciences, 18, 51 - 66.

Hamedani, G. G. (2013), On certain generalized gamma convolution distributions II. Technical Report, 484, MSCS, Marquette University.

Hosking, J. R. M. (1990), L-moments: analysis and estimation of distributions using linear combinations of order statistics. Journal of the Royal Statistical Society. Series B (Methodological), 52, 105 - 124.

Kandil, A. E. F. M., Kayid, M., and Mahdy, M. M. R. (2010), Median inactivity time function and its reliability properties. Economic Quality Control, 25(2), 253 - 268.

Khan, A. H., and Alzaid, A. A. (2004), Characterization of distributions through linear regression of non-adjacent generalized order statistics. Journal of Applied Statistical Science, 13, 123 - 136.

Khan, A. H., Faizan, M., and Haque, Z. (2010), Characterization of continuous distributions through record statistics. Communications of the Korean Mathematical Society, 25 (3), 485-489

Kumar, D. (2017), The Burr type XII distribution with some statistical properties. Journal of Data Science, 15(3), 509 - 533.

Mdlongwa, P., Oluyede, B., Amey, A., and Huang, S. (2017), The Burr XII modified Weibull distribution: model, properties and applications. Electronic Journal of Applied Statistical Analysis, 10(1), 118 - 145. 
Mead, M. E., and Afify, A. Z. (2017), On five-parameter Burr XII distribution: properties and applications. South African Statistical Journal, 51, 67 - 80.

Muhammad, M. (2016), A generalization of the Burr XII-Poisson distribution and its applications. Journal of Statistics Applications and Probability, 5(1), 29 - 41.

Nagarajah, H. (1988), Some characterizations of continuous distributions based on regressions of adjacent order statistics and record values. Sankhya Series A, 50, 70 - 73.

Okasha, M. K. and Matter, M. Y. (2015), On the Three-Parameter Burr Type Xii Distribution and its Application to Heavy Tailed Lifetime Data. Journal of Advances in Mathematics, 10, 3429 - 3442.

Olapade, A. K. (2008), On a six-parameter generalized Burr XII distribution. arXiv preprint arXiv:0806.1579s

Paranaíba, P. F., Ortega, E. M., Cordeiro, G. M. and Pescim, R. R. (2011), The beta Burr XII distribution with application to lifetime data. Computational Statistics $\mathcal{E}$ Data Analysis, 55, 1118 - 1136.

Paranaíba, P. F., Ortega, E. M., Cordeiro, G. M. and Pascoa, M. A. D. (2013), The Kumaraswamy Burr XII distribution: Theory and Practice. Journal of Statistical Computation and Simulation, 83, 2117 - 2143.

Thupeng, W. M. (2016), Use of the Three-parameter Burr XII Distribution for Modelling Ambient Daily Maximum Nitrogen Dioxide Concentrations in the Gaborone Fire Brigade. American Scientific Research Journal for Engineering, Technology, and Sciences (ASRJETS), 26, 18 - 32.

Saran, J. and Pushkarna, N. (1999), Moments of order statistics from doubly truncated Lomax distribution. Journal of Statistical Research, 33, 57-66.

Shannon, C. (1948), A mathematical theory of communication. Bell System Technical Journal, 27, 379 - 423.

Shao, Q., Wong, H., Xia, J. and Ip, W. C. (2004), Models for extremes using the extended three-parameter Burr XII system with application to flood frequency analysis. Hydrological Sciences Journal, 49, 685 - 701.

Silva, R. B. and Cordeiro, G. M. (2015), The Burr XII power series distributions: A new compounding family. Brazilian Journal of Probability and Statistics, 29, 565 - 589. 
Tadikamalla, P. R. (1980), A look at the Burr and related distributions. International Statistical Review/Revue Internationale de Statistique, 48, 337 - 344.

Takahasi, K. (1965), Note on the Multivariate Burr's Distribution. Annals of the Institute of Statistical Mathematics, 17, 257 - 260.

Usta, I. (2013), Different estimation methods for the parameters of the extended Burr XII distribution. Journal of Applied Statistics, 40, 397 - 414.

Yari, G., and Tondpour, Z. (2017), The new Burr distribution and its application. Mathematical Sciences, 11, 47 - 54.

Zimmer, W. J. and Burr, I. W. (1963), Variables sampling plans based on non-normal populations. Sandia Corporation.

\section{Appendix A}

Theorem 9.1. Let $(\Omega, F, P)$ be the probability space and $H=\left[a_{1}, a_{2}\right]$ be an interval with $a_{1}<a_{2}\left(a_{1}=-\infty, a_{2}=\infty\right)$. Let $X: \Omega \rightarrow\left[a_{1}, a_{2}\right]$ be a continuous random variable with the distribution function $F$ and let $q$ be a real function defined on $H=\left[a_{1}, a_{2}\right]$ such that $E[q(X) \mid X \geq$ $x]=\eta(x), x \in H$, is defined with some real function $\eta(x)$. Assume that $q(x) \varepsilon C\left(\left[a_{1}, a_{2}\right]\right)$, $\eta(x) \varepsilon C^{2}\left(\left[a_{1}, a_{2}\right]\right)$ and $F$ is a twofold continuously differentiable and strictly monotone function on the set $\left[a_{1}, a_{2}\right]$. Assume that the equation $q(x)=\eta(x)$ has no real solution in $\left(a_{1}, a_{2}\right)$. Then, $F$ is obtained from the functions $q(x)$ and $\eta(x)$ as

$$
F(x)=\int_{a}^{x} k\left|\frac{\eta^{\prime}(t)}{\eta(t)-q(t)}\right| \exp (-s(t)) d t
$$

where $s(t)$ is the solution of the equation $s^{\prime}(t)=\frac{\eta^{\prime}(t)}{\eta(t)-q(t)}$ and $k$ is a constant, chosen to make $\int_{a_{1}}^{a_{2}} d F=1$ 
